\title{
ON THE NATURE OF THE X-RAY SOURCES SCORPIUS X-1 AND CENTAURUS X-2*
}

\author{
SABATINO SOFIA \\ Dept. of Astronomy, University of South Florida, Tampa, Fla., U.S.A.
}

\begin{abstract}
Preliminary evidence is presented which indicates that the star WX Cen, associated with the X-ray source Centaurus X-2 belongs to the Scorpio-Centaurus association. It is pointed out that this result greatly strengthens the similar results for the source Scorpius X-1, and lends support to the idea of the existence of a class of X-ray stars formed by young, subluminous objects, probably supernova remnants, thus neutron stars.
\end{abstract}

On carrying out an accurate proper motion study (by the plate overlap technique) of 83 stars in the region surrounding the optical object associated with the X-ray source Sco X-1, it was found that Sco X-1 together with at least three more of these stars have proper motions very similar to the nearby B stars which are well known members of the Scorpio-Centaurus association (Sofia et al., 1969). Following the general practice in such cases, we concluded that Sco X-1 is probably a member of the association. Thus, the accurate distance to the source is about 200 parsecs, and the upper limit for its age is 20 million years (cf. Bertiau, 1958). We soon realized (Gatewood and Sofia, 1968) that the resulting source parameters impose some severe constraints on the nature of the X-ray star. In particular, since Sco X-1 appears substantially below the main sequence (see Figure 2 of Gatewood and Sofia's paper), according to well established results in stellar evolution, the object must have evolved off the main sequence in less than 20 million years. This means (Iben, 1967) that the main sequence mass of the object must have been above $10 \mathrm{M}_{\odot}$, and according to Colgate and White (1966), an object that massive would have undergone a supernova explosion and probably now be a neutron star.

The idea of a relationship between X-ray sources and neutron stars is not new. Soon after the first detection of X-ray sources, Chiu and Salpeter (1964) proposed that they were hot neutron stars. The observed characteristics of Sco X-1 are, however, very different from those expected according to the early picture of the X-ray emitting neutron stars. In particular, the spectrum (from the X-ray to the visual region) requires most of the radiation to originate in a cloud which surrounds the neutron star, and has a radius of at least a hundred times that of the star (cf. Shklovsky, 1967).

In view of the implications of these results, much effort was spent in checking the accuracy of the method. For example, it was easy to verify that stars \#13 and \#25 in the catalogue in the paper by Sofia et al., for which spectral classification was available, perfectly fit the main sequence once corrected for a distance modulus of

* Astronomical Contributions from the University of South Florida at Tampa, No. 23. 
$6.1 \mathrm{mag}$. and an absorption of $0.1 \mathrm{mag}$., corresponding to the values derived for the Scorpio-Centaurus association by Bertiau (1958). The same happened for the star \# 37 which was classified GOV from a spectrum taken by J. Hunter at the Yale 40 inch telescope. It is well known, however, that the main weakness of any proper motion result is the ever present possibility that, through a series of coincidences, a star not belonging to a certain group would have the exact tangential component of its peculiar velocity (both in direction and magnitude) to offset distance differences so as to appear to move within the group. I am now pleased to report that a preliminary study indicates that the star WX Cen, recently identified with the source Cen X-2 (Eggen et al., 1968) is also a member of the Scorpio-Centaurus association. Its location in the H-R diagram very near Sco X-1 appears to indicate both that the identification of Cen X-2 is correct, and that the results for Sco X-1 are real, and not the consequence of possible freak coincidences pointed out above.

In a work performed at the University of South Florida in collaboration with H. Eichhorn and G. Gatewood, we determined the positions and proper motions of 124 stars in the region of Cen X-2, including the star WX Cen. The early epoch plate (1897) was kindly provided to us by Mr. Harley Wood, of the Sydney Observatory, while the recent epoch plates were taken for us by Mr. G. A. Harding at the Cape Observatory in South Africa. The position and proper motion of Cen X-2 in the $\mathrm{FK}_{4}$ system are

$\begin{array}{cccc}\alpha_{1950} & \mu & \delta_{1950} & \mu^{\prime} \\ 13^{\mathrm{h}} 9^{\mathrm{m}} 38^{\mathrm{s}} .11 & -0.0030 & -63^{\circ} 7^{\prime} 51^{\prime \prime} 3 & -0^{\prime \prime} 004 \\ \pm 0.030 & \pm .00090 & \pm .16 & \pm .0056\end{array}$

The errors are rms errors.

At least five other stars with photographic magnitudes ranging from 7.8 to 14 have been found to have proper motions similar to that of Cen X-2. The only one for which we have a spectrum, CPD $-63^{\circ} 2636$, fits the main sequence of the Scorpio-Centaurus association which in this region has a distance modulus of $6.3 \mathrm{mag}$. (Bertiau, 1958). The above proper motion may be compared with that of the nearest member in Bertiau's list, namely HD 116087, whose proper motion components $\left(\mu, \mu^{\prime}\right)$ in the system of the $\mathrm{FK}_{4}$ are $(-0.0034 \pm .00057,-0.011 \pm .0041)$ respectively, the errors being rms errors.

It is in summary concluded that, if additional checks confirm this preliminary result, substantial evidence exists which indicates the existence of a class of X-ray stars, of which Sco X-1 and Cen X-2 are members, consisting of young, subluminous objects which probably are supernova remnants, and thus according to current ideas, neutron stars.

\section{Acknowledgement}

The work reported has been supported by the NSF Grant No. GP 8121, for which the author is very grateful. 


\section{References}

Bertiau, F. C.: 1958, Astrophys. J. 128, 533.

Chiu, H. Y. and Salpeter, E. E.: 1964, Phys. Rev. Letters 15, 599.

Colgate, S. A. and White, R. H.: 1966, Astrophys. J. 143, 626.

Eggen, O. J., Freeman, K. C., and Sandage, A., 1968, Astrophys. J. 154, L27.

Gatewood, G. and Sofia, S.: 1968, Astrophys. J. 154, L69.

Iben, I.: 1967, Ann. Rev. Astron. Astrophys. 5, 571.

Shklovsky, I. S.: 1967, Astrophys. J. 148, L1.

Sofia, S., Eichhorn, H., and Gatewood, G.: 1969, Astron. J. 74, 20. 